Revue d'histoire de l'Amérique française

REVUE D.HISTOIRE DE L'AMÉRIQUE FRANÇAISE

\title{
Un témoignage de 1651 sur la Nouvelle-France
}

\section{Lucien Campeau}

Volume 23, numéro 4, mars 1970

URI : https://id.erudit.org/iderudit/302936ar

DOI : https://doi.org/10.7202/302936ar

Aller au sommaire du numéro

Éditeur(s)

Institut d'histoire de l'Amérique française

ISSN

0035-2357 (imprimé)

1492-1383 (numérique)

Découvrir la revue

Citer ce document

Campeau, L. (1970). Un témoignage de 1651 sur la Nouvelle-France. Revue

d'histoire de l'Amérique française, 23(4), 601-612.

https://doi.org/10.7202/302936ar d'utilisation que vous pouvez consulter en ligne.

https://apropos.erudit.org/fr/usagers/politique-dutilisation/ 


\section{DOCUMENTS INÉDITS}

\section{UN TÉMOIGNAGE DE 1651 SUR LA NOUVELLE-FRANCE}

Le document que nous présentons ici ne semble pas avoir été utilisé comme source de notre histoire. Nous l'avons trouvé aux archives de la S.C. de la Propagande, à Rome, dans Scritture originali riferite nelle Congregazioni generali, vol. 260, ff. 310313. C'est un manuscrit du dix-septième siècle, comportant quatre feuillets de $0,310 \times 0,215 \mathrm{~m}$. Le papier est bruni par le temps. Le texte occupe les deux faces des trois premiers feuillets, le quatrième demeurant vierge. En haut de la première page, cette note d'un secrétaire: "Lettere libere $1652 \mathrm{f}^{\circ} 5^{\circ}$ ". Cette année 1652 est sans doute celle où le document a été classé pour la première fois. Autre note, en bas de la même page: "Indie occidentali, Quebeko". Un ancien numéro du document, 223, a été raturé, de même qu'une ancienne numération des folios: 286-289.

Ce n'est ni un autographe ni un original. L'en-tête affirme d'ailleurs que c'est une copie. Ou mieux, c'est une traduction d'une lettre qui a vraisemblablement été rédigée en français. Le latin conserve d'ailleurs le caractère plus primesautier de notre langue.

Nous attribuons cette lettre à Simon Denys, qui s'établit à Québec en 1651. Son arrivée est attestée par le Journal des Jésuites ${ }^{1}$, à la date du 12 octobre de cette année. Les "Messieurs Denys" qui arrivent alors en compagnie du gouverneur Jean de Lauzon, délivrés d'une captivité infligée par madame d'AunayCharnisay en Acadie, sont, à n'en pas douter, les deux frères, Nicolas et Simon. Le premier sera gouverneur et lieutenant du Roi en Acadie et auteur de deux précieux volumes sur cette région de la Nouvelle-France ${ }^{2}$. Simon Denys laissera une nom-

${ }^{1}$ Laverdière et Casgrain, éd., Le Journal des Jésuites, $2 \mathrm{e}$ édition, (Montréal, 1892), 162.

2 Nicolas Denys, Description géographique et historique des costes de l'Amérique septentrionale, avec l'Histoire naturelle du Païs (2 tomes, Paris, M. DC. LXXII). Réédité par William F. Ganong, The Description and Natural History of the Coasts of North America (Acadia) by Nicolas Denys, (Toronto, The Champlain Society, 1908), texte français et traduction anglaise.

[601]

Vol. XxIII, no 4 (mars 1970) 
breuse et remarquable postérité dans la colonie du Saint-Laurent. Que ce dernier soit l'auteur de notre document, cela est indiqué par l'intention, déclarée dans le texte, de faire un établissement à Québec, ce que Simon a exécuté par la suite. Le nom du destinataire de la lettre, Du Breuil, que l'auteur appelle aussi son frère, le confirme encore. Simon Denys avait épousé en premières noces Jeanne Du Breuil, qui était morte vers 1639 à Tours. Le sieur $\mathrm{Du}$ Breuil est donc proprement le beau-frère de Simon Denys.

Il n'y a pas lieu de faire ici la biographie de Simon Denys, qu'on trouvera dans le Dictionnaire biographique du Canada ${ }^{3}$ et dans le Dictionnaire général ${ }^{4}$ du P. Lejeune. L'une et l'autre de ces œuvres a cependant besoin d'être complétée par les notes de W.F. Ganong ${ }^{5}$. Nous ferons seulement ici quelques remarques. Nicolas Denys semble donner à Simon le titre de sieur de Vitray ${ }^{6}$. Cette appellation sera en fait portée par le fils de ce dernier, Charles, sous la forme Vitré. Il n'est pas sûr que Simon ait eu ce titre dès son arrivée à Québec; peut-être ne l'a-t-il porté qu'à la fin de sa vie, du moins après 1668, année où il obtint des lettres de noblesse ? . De son vivant, il a été plus souvent appelé sieur de La Trinité, terre qu'il reçut des Jésuites en 1652 dans la seigneurie de Notre-Dame-des-Anges ${ }^{8}$. Contrairement à ce qu'en a dit le P. Hugolin Lemay ${ }^{9}$, cette terre n'était pas un fief noble; on ne la voit pas comptée parmi les seigneuries. Le P. Lejeune se trompe en l'identifiant avec l'emplacement de la citadelle de Québec, identification qui est d'ailleurs invraisemblable. Et elle n'est pas à confondre avec le fief de Montapeine, concédé plus tard à Charles Denys de Vitré et situé sur la côte de Lauzon. Simon Denys sera l'un de ceux que le gouverneur Saffray de Mézy nommera au Conseil souverain, le 4 septembre 1664, passant outre aux prérogatives de Mgr de Laval ${ }^{10}$. Cette

3 Dictionnaire biographique du Canada, vol. I, Les Presses de l'Université Laval [Québec, 1965], 269.

4 Louis Lejeune, Dictionnaire général de biographie, histoire, littérature ... du Canada (2 tomes, Ottawa [1931]), I: 493-494.

5 Ganong, The Description ..., introduction et 143n, 183-184n.

6 Ibid., 480.

7 Bulletin des Recherches historiques (Lévis, 1906), 12: 345-346.

8 P.-G. Roy, Inventaire des concessions en fief et seigneurie (Beauceville, 1927), I: 27.

9 Hugolin Lemay, Le Père Joseph Denis, Premier Récollet canadien (1657-1736) (Québec, 1926), II : 173. I: 281 .

10 Jugements et Délibérations du Conseil souverain (Québec, 1885), 
charge cessera avec la restauration du Conseil par le marquis de Tracy le 6 décembre $1666^{11}$.

Notre document ajoute quelques informations inédites sur le personnage. On apprend qu'il était le premier-né des frères Denys, alors que tous les auteurs attribuent cet avantage à Nicolas. En fait, on ignore les dates de naissance pour les deux. Notre source, qui est contemporaine, paraît apporter un témoignage décisif sur ce point. Ainsi, s'il est vrai, comme on l'affirme, que Nicolas est né en 1598, Simon a dû voir le jour auparavant. Il mourut entre 1678 et 1680 .

Sur l'arrivée à Québec de Françoise du Tartre, la seconde femme de Denys, épousée en 1645 , notre document apporte encore un témoignage non équivoque. Elle accompagnait son mari en octobre 1651 . C'est dire qu'elle habitait déjà l'Acadie avant cette date et qu'elle a partagé la captivité de son mari. Probablement aussi les enfants de Simon Denys: ceux du premier lit, Pierre, Anne, Charles et Françoise, et ceux du second, Catherine, Paul et Marguerite. Cette dernière, née en 1651, dut voir le jour en Acadie, non en France.

Ainsi, l'on comprend l'allusion faite par Denys à la facilité de marier les filles en Nouvelle-France. Anne, qui épousera Louis Chartier de La Broquerie ${ }^{12}$, était déjà d'âge nubile et la famille comptait trois autres filles.

On pourra s'étonner qu'à seize jours de son arrivée à Québec Simon Denys ait pu formuler un jugement bien appuyé et objectif sur la colonie du Saint-Laurent. Il ne faudrait pas oublier qu'il n'est pas un nouveau venu en Amérique du Nord, puisqu'il fréquente l'Acadie depuis 1632 en compagnie de Nicolas. Capitaine de vaisseau, Simon a probablement déjà remonté le SaintLaurent. Les deux Denys, exploitant le commerce du golfe, ne pouvaient guère éviter d'entrer en rapport avec Québec. C'est ce que laissent entendre les expressions du Journal des Jésuites à leur sujet. Leur arrivée de 1651 est présentée comme un retour: "furent aussi renvoyez", "Msr Denys de retour". Simon avait sans doute une connaissance préalable de la colonie. Ce n'est donc pas un métropolitain frais arrivé qui écrit, mais plutôt un habitué de l'Acadie passant dans la colonie voisine, mieux structurée et plus tranquille.

Nous avons cru indispensable de présenter le texte latin dans son intégrité. Mais il était opportun, pensons-nous, d'en faire la

11 Ibid., 367.

12 Nova Francia (Paris, 1926-1927), II : 35 et 138. 
traduction. Les deux seront mis en regard et livrés au choix du lecteur. La division en paragraphes est de nous.

Département d'histoire

LUCIEN CAMPEAU, s.j.

Université de Montréal

\section{Copie d'une lettre du sieur Denys l'aîné, de la ville de Québec, 28 octobre 1651}

1. Le Dieu très bon semble me donner une vigueur nouvelle pour travailler dans ce pays. En France, j'étais toujours malade et si faible que je pouvais à peine demeurer une heure sur pied; à présent, une journée entière ne suffit pas à me fatiguer. Je ne pouvais prendre qu'un peu de vin coupé d'eau; aujourd'hui, je bois le vin pur et l'eau-de-vie sans incommodité. Rien d'étonnant à cela, car tous jouissent d'une bonne santé semblable. Monsieur de Lauzon, notre gouverneur, qui ne sortait pas à Paris autrement qu'en calèche, marche ici bien à l'aise et il mange sans inconvénient de toute nourriture, malgré ses soixante-dix ans. Dans ce pays, on ne sait ce qu'est l'arthrite, la fièvre et le catarrhe. De médecins et de pharmaciens, nul besoin; pour toute la ville de Québec, un seul chirurgien suffit ${ }^{13}$.

Exscriptum epistolæ domini Dionysii primogeniti, ex urbe Quebecco, $28^{\circ}$ octobris 1651

1. Deus optimus vires mihi novas ad operandum in hac regione videtur subministrare. In Gallia semper ægritudine confectus atque adeo debilis, ut vix una hora in pedes stare potuissem; nunc vero dies ad me fatigandum brevior est. Aquam nonnisi vino modico dilutam poteram ebibere; nunc merum et aquam vitæ absque ullo mei dispendio bibo. Nec mirum. Omnes etenim eadem utuntur valetudine. Dominus de Lauzon, noster præfectus, qui Parisiis nonnisi carpento vehebatur,

10 quamvis septenarius, optime omnium pedibus incedit ac ex omnibus cibis non fastidiose comedit. In hac regione arthritides, febres atque catarrhi ignorantur. Medicis ac pharmacopolis hic non opus est. Sufficit unus pro tota urbe Quebecco chirurgus.

2. Ce n'est pas la seule bénédiction dont Dieu a favorisé ce pays. Il faut le voir pour le croire. Le sol est fertile et produit la

13 Il est difficile de dire qui était ce chirurgien. On en connaît trois qui ont résidé en Nouvelle-France à cette époque: Adrien Duchesne, François Gendron et Jean Madry. Ce dernier, mentionné au recensement de 1666, n'était probablement pas encore au pays en 1651. François Gendron, chirurgien chez les Hurons, est rentré en France en 1650. Reste Adrien Duchesne, oncle de Charles Le Moyne, dont on n'a pas d'assurance qu'il ait encore demeuré à Québec en 1651. C'est toutefois possible. 
meilleure qualité de blé. Les semailles se font en octobre et la récolte en août; ou encore on sème en mai et on moissonne en septembre. Les choux et les navets viennent ici à merveille; les citrouilles sont beaucoup plus savoureuses qu'en France. Les enfants les cuisent sous la cendre et s'en font un plat parfaitement délicieux. Toutes les légumes croissent facilement. Le blé d'Inde, vendu aux sauvages, rapporte un profit très élevé. Les pois et les fèves poussent ici en abondance. Les eaux sont des plus saines, mais la bière encore bien plus. Et je ne doute pas que le vin n'y soit aussi des meilleurs. J'ai vu des raisins blancs et noirs arriver à une maturité parfaite. Les melons sont bons pour la plupart; j'en ai quelquefois mangé d'exquis.

2. Non hac unica benedictione Deus regioni huic favet. Nisi 15 cerneretur, credi id non posset. Tellus fœcunda est ac exquisitissimum fert triticum. Seritur octobri et augusto metitur; aliud maio seritur et septembri colligitur. Caules et napi hic stupendi; citrinæ cucurbitæ longe sapidiores sunt quam in Gallia. Pueri eas sub cineribus concoquere solent et inde optimum sibi componunt obsonium. Omnia olera

20 facillime succrescunt. Frumentum indicum ad commutandum cum sylvicolis maximum affert emoluentum. Pisa et fabæ ibi abunde crescunt. Aquæ saluberrimæ sunt; salubrius adhuc zythum. Nec dubito [310v] quin vinum esset hic peroptimum. Racemos vidi albos et nigros ad perfectam devenisse maturitatem; melones, ut plurimum boni. Ego exquisitissimos quandoque comedi.

24 ut... boni corr. sur ligne d'illis.

3. Le pays abonde en poissons, surtout en saumons, mais aussi en turbots, rougets et esturgeons. Les truites, de même que les anguilles, $\mathrm{y}$ foisonnent. D'aucuns saleront vingt ou trente barils d'anguilles pêchées devant leur porte. Ce poisson, dans ce pays, tient lieu de la viande de bœuf et l'on s'en nourrit durant toute l'année sans en être dégoûté. Il est excellent, frais ou salé. Il n'a pas besoin de beurre et donne une graisse abondante, qui sert à faire des sauces pleines de saveur et dont on ne se fatigue pas. Plusieurs préfèrent l'anguille à la chair des oiseaux que l'on prend ici en grand nombre.

3. Piscibus abundat, præsertim salmonibus, sicut et rhombis, mullis et acipenseribus. Truttæ, sicut et anguillæ, illic affluunt. Quidam sunt qui salient viginti aut triginta cadi anguillarum quas præ foribus suis expiscantur. Piscis ille pro huius regionis carne bovina

30 habetur; eo per totum annum absque fastidio vescuntur. Recentis et sale conditi sapor est eximius; butyro et condimento non indiget; sat adipis effundit, ex quo optima iuscula conficiuntur quæ nusquam fastidium procreant. Multi anguillam præferunt aviariæ prædæ, quæ hic plurima est.

\section{Quidam sunt corr. sur ligne d'illis.}


4. En quelques îles et en quelques endroits, il y a telle abondance de ces oiseaux qu'on en remplit plusieurs barques, surtout d'oies blanches, d'outardes, de sarcelles, d'alouettes et de canards. Ils s'y posent en troupes si compactes et si nombreuses qu'ils couvrent des surfaces très étendues. Les tourtes y nichent en bandes durant tout l'été, tandis que les perdrix y abondent en hiver.

4. Sunt insulæ et loca quædam in quibus tanta est avium copia ut eis plures cymbæ onerentur, præcipue anserum alborum, otidum, querquedularum, pluvialium, alaudarum et anatum. Adeo condensi greges illuc advolant, ut ingentia soli spatia cooperiant. Turtures more 40 columbarum illic catervatim per totam æstatem degunt; perdicibus vero hyeme abundant.

5. Le lièvre, le castor, l'ours, l'orignal se rencontrent partout. Près du port des Trois-Rivières broutent des troupeaux de vaches portant des bois au lieu de cornes, à la manière des cerfs ${ }^{14}$. Les familles ont un nombre étonnant de bovins, de dindes et de poules.

5. Lepus, castor, ursus, orignacus ubique reperiuntur. Iuxta portum Trium Fluminum armenta vaccarum pascuntur quæ ramosa cervorum cornua gestant. In familiis, boves, porci, meleagrides et gallinæ mirum in modum pullulant.

6. Les prairies ne coûtent qu'à faucher et engranger. La forêt présente des inconvénients l'été, mais elle a beaucoup d'avantages l'hiver. Le feu brûle jour et nuit à pleine cheminée. Dans les champs, on entasse le bois et l'on y met le feu. L'espoir du profit rend les hommes habiles et laborieux. En toute saison de l'année l'on a à faire. En hiver, on coupe les bois pour étendre l'espace cultivé; au printemps, on les brûle; puis on fait les semailles. Un homme actif peut très facilement essarter et semer deux arpents, qui produiront pour leur maître de quoi bien vivre au bout d'une année. En sorte qu'on est entièrement hors de besoin; on vit avec aise et agrément.

6. Prata nihili constant nisi in demetendo et recondendo. Sylvæ incommodum æstate, hyeme vero commodum afferunt plurimum. Ignis die ac nocte pleno accenditur camino. Adde quod in campis acervatim comburitur. Emolumentum peritos ac expeditos efficit. Quavis anni tempestate operi incumbunt. Hyeme sylvæ ad novandos agros resecantur; vere, illas comburunt; deinde agros conserunt. Vir expeditus duo iugera novare atque conserere facillime potest, quæ domino suo

${ }^{14}$ Ce sont des caribous, qui vivent en bandes et dont les femelles portent des bois comme les mâles. 
victum in primum annum abunde suppeditent. [311] Hinc egestas omnis exulat, bene beateque vivitur.

7. Personne n'est si à court de moyens qu'il ne soit en mesure de tuer deux porcs engraissés pour sa nourriture et pour l'achat aux sauvages d'un lot de castors de sept à huit cents écus. On demandait à une femme du commun à combien se montait une vente semblable qu'elle avait faite: "Seulement à mille cinq cents livres", répondit-elle. Toutefois, on ne vend d'ordinaire que le blé d'Inde, le pain, les pois et les citrouilles.

7. Res familiaris nulli adeo curta et tenuis est quin duos pingues 55 et saginatos in victum porcos occidat atque cum sylvicolis septingentis aut octingentis nummis castores merceatur. Mulier quædam mediocris quærentibus quanti negotiata fuerat: "Mille et quinquentis tantum, respondit, nummis". Attamen ea tantum in merce sunt: frumentum nempe indicum, panis, pisa citrinæ cucurbitæ.

8. L'usage est ici de ne passer en voyage devant aucune habitation, même de condition moyenne, sans être invité à entrer, à boire du lait, même à déguster le vin, s'il s'en trouve, et aussi sans qu'on vous mette la table, si l'on a de quoi manger. Un noble serait offensé qu'on passe sa maison sans la visiter. Ils se visitent les uns les autres comme le feraient des frères. Tous sont nobles, libres de taxes, de tributs, d'emprunts, de réquisitions militaires et d'autres exactions quelconques.

60 8. Mos hic inolevit ut domum vel mediocris non possis prætergredi quin te invitet ut tecto succedas, lac comedas, tum etiam, si vinum suppetit, propinet, et cibos, si quos habet alios, apponat. Dedecori ducat maximo vir nobilis, si pertransiens eum ex itinere non invisas. Alter alterum non secus ac fratres invisunt se mutuo; omnes sunt nobiles et vectigalibus, tributis, mutuationibus, militibus et exactionibus quibuslibet immunes.

9. La terre ne manque pas. Les maisons ne sont pas verrouillées contre les voleurs, que l'on connaît à peine de nom. Les procès se règlent sans délais, sans frais et sans dépens. On n'entend pas parler de querelles; de voies de fait, nulle mention; pas de blasphèmes. Lorsqu'une dispute s'élève, on n'attend pas que le grief s'enfle jusqu'à l'injure. Les Pères de la Compagnie de Jésus apaisent tous les soulèvements avec tant de prudence que tous les honorent comme des saints et des oracles, que tous les écoutent et les aiment. Notre Gouverneur interdit à ses serviteurs d'empêcher qui que ce soit de l'approcher, afin que personne n'ait sujet de se dire lésé. Il veut que sa porte soit ouverte à tous; il les écoute avec une patience admirable et donne satisfaction à chacun. En somme, personne n'a jamais décrit l'âge d'or avec autant d'éclat qu'on le voit réalisé et manifesté dans ce pays. 
Les mœurs sont polies; la langue française y est parlée avec élégance. Hommes, femmes et enfants, si la famille a quelque naissance, s'habillent et s'ornent avec non moins de propreté et de grâce qu'on ne le fait à Paris.

9. Nulla est terræ parcitas. Ne latrones, qui vix de nomine noti sunt, subrepant, ædes non occluduntur. Lites ex tempore dirimuntur, sine sumpsibus, nullis impensis; nulla audiuntur dissidia; iurgiorum 70 nulla mentio, blasphemiæ nullæ. Ubi primum quid contentionis emersit, quo ad iniuriam conquestum veniant non exspectatur. Omnes motus tanta prudentia Patres Societatis Iesu compescunt, ut ab omnibus æque ac sancti atque oracula colantur, observentur, diligantur. Præfectus noster suis vetat ne quemquam a suo congressu arceant, ne se dicant esse impeditum; vult omnibus liberum ad se aditum patere. Omnes mira æquanimitate audit; cuique satisfacit. Ad summum, nullus adeo solerter ætatem auream depinxit unquam ut se ipsa hac in regione prodit et exhibet. Sunt urbani mores; non inconcinnus gallicæ linguæ viget usus. Viri et mulieres eorumque filii, quicumque sunt illustri loco

80 nati, veste utuntur et cultu haud deteriore nec inferiore eo qui Parisiis in usu est.

80. veste corr. sur ligne de cultu / cultu corr. sur ligne de veste

10. Québec ressemble et ne cède en rien à toute autre ville de France, même au regard des édifices religieux et de leur riche ameublement. On y pratique la piété sans fard et tous ont une sorte d'inclination naturelle aux exercices de charité. Je l'ai éprouvé moi-même par expérience. Dès mon arrivée, tous se disputèrent l'honneur de me faire accueil. Tous les notables nous firent visite, montrant pour notre malheur une compassion si grande et si profonde qu'ils n'auraient pu en témoigner davantage pour une disgrâce arrivée à leurs parents. Si leur bonne volonté n'eût été aussi sincère et évidente, leur serviabilité m'aurait même gêné. Ils me recurent et me fournirent le manger dont $\mathrm{j}$ 'avais besoin, une petite maison où demeurer jusqu'à ce que j'eusse trouvé un domicile plus convenable et plus commode. Ils se chargèrent d'y porter tous mes meubles. Même les paysans me donnèrent le témoignage de leur intérêt: ils nous offrirent des choux, du beurre, du lait, des navets, des prunes, des poulets. En sorte que, par la grâce de Dieu, il ne nous manque que du vin et de l'eau-de-vie, que l'on m'a promis, avec des anguilles, contre de l'argent. Notre Gouverneur m'a offert de manger à sa table. Ici, la condition des pauvres est heureuse. Nul besoin de mendier. Au contraire, tout vous est offert avec tant de générosité qu'on paraît faire une grâce en acceptant les dons.

10. Quebecum simile est nec ulla in re cedens ulli Galliæ urbi, nec ipsæ etiam sacræ ædes et earum opulenta supellex. Pietas colitur absque fuco et omnes ad obeunda charitatis officia ab ipsa natura 
85 proclives sunt. Cuius ego singularis in me ipsum argumentum expertus sum. Vix enim adveneram, cum omnes de me excipiendo quasi inter se certarunt. [311v] Primores omnes inviserunt nos, tantum ob iacturam nostram dolorem præ se ferentes, ut nostra quasi suorum cognatorum iniuria intimis sensibus affecti viderentur. Nisi magna quædam et eximia

90 eorum benevolentia innotuisset, eorum mihi fuissent officia molesta. Exceperunt me et quæ ad victum necessaria sunt subministrarunt, ædiculam in qua habitarem, quoad oportunius et commodius domicilium nactus sim, assignarunt. Omnem meam supellectilem convehendam curarunt. Vel ipsi agrestes suum in nos studium significarunt; nobis 95 caules, butyrum, lac, napos, pruna, pullos gallinaceos obtulerunt; sic prorsus ut, quæ Dei gratia est, solo vino et aqua vitali careamus, quæ mihi cum anguillis pro pecunia promissa sunt. Oblata mihi est Præfecti nostri mensa. Felix est hic pauperum conditio. Nihil emendicandum est; quin e contrario tam benevolo animo offerunt, ut in se 100 collatum beneficium arbitrentur si quæ offerunt excipiantur.

87 certarunt corr. de certabant // 94 apr. ipsi rat. etiam

11. Lorsqu'en France vous entendez parler du Canada, vous imaginez un désert inculte et plein d'horreur. Descendant de Québec, on voit les maisons des habitants disséminées sur une longueur de sept lieues et distantes les unes des autres d'une portée d'arquebuse environ. Et en remontant le fleuve, sur une longueur de deux lieues, l'œil ne s'arrête que sur des champs défrichés. La terre est légère et noire comme de l'encre.

11. Si quid in Gallia de Canadia audis, tibi fingis animo horrentem quamdam et incultam solitudinem. Ex Quebecco descendentibus per septem leucas passim incolarum ædes occurrunt a se invicem tanto forte intervallo dissitæ quantum globulus explosa catapulta emissus 105 pervolet. Iam vero a superiori parte qua amnis defluit, per duas leucas, ubi quantum se acies intendant camporum prospectus aperitur. Quorum solum leve est et atramentum nigredine referens.

12. On sème tous les ans et s'il n'y avait nombre de sauvages, qui ne font rien que chasser et manger, le minot de blé, vendu aujourd'hui à cinquante sols, en coûterait à peine cinq. Pour six livres de pain, on achète du castor ou une peau d'orignac. Un ouvrier gagne chaque jour cinquante ou soixante sols. C'est pourquoi celui qui voudrait habiter ici serait très bien avisé d'en emmener de France au salaire auquel on les engage là-bas. Trois années durant, ils travailleraient pour lui. Ce temps expiré, ils seraient libres de retourner en France ou d'élire domicile en ce pays. Quant à se mettre au service d'un autre maître, cela ne leur est permis qu'avec l'agrément de leur patron. Même sans de telles ententes, chacun trouve facilement de quoi manger.

12. Seritur quotannis et, nisi magna obesset sylvicolarum frequentia, qui venantur tantummodo et comedunt, tritici modius qui 
110 modo quinquaginta vænit assibus, tum vix quinque constaret. Sex libris panis castorem emes aut pellem orignaci. Operarius 50 aut 60 assos quotidie lucrifacit. Quocirca qui hic volet habitare suis rebus optime consuleret si ex Francia adveheret eadem qua eos conduxeris mercede. Tibi operam navabunt suam per tres annos. Quo tempore 115 expleto ipsis liberum est et integrum in Galliam reverti aut suum inibi domicilium stabilire. Aliis vero operam suam addicere, nisi te probante, ipsis non licet. Absque eo pacto quisque facile et commode victum quærit.

13. Il y a ici un prêtre qui nous dit chaque jour la messe ${ }^{15}$. Il n'a pas de revenu ni d'honoraires réguliers. Cependant, il reçoit magnifiquement tous ses visiteurs. Il gagne sa vie à tisser des rêts, dont on fait grand usage pour la pêche; et cette industrie lui rapporte plus que ne ferait en France aucun des principaux bénéfices curiaux. Il nous a fait cadeau de vingt-cinq gros choux feuillus, bien meilleurs que ceux que produit la France. Son voisin nous a donné trois minots de navets. Et d'autres ont fait de même.

13. Sacerdos hic est qui nobis singulis diebus sacra facit. Nullos 120 habet reditus; nulla stata stipendia. Nihilominus, quotquot eum invisunt omnes laute excipit. [312] Contexendis retibus, quorum plurimus ad capiendos pisces usus est, victum parat; hæcque sua industria plus illi affert emolumenti quam in Gallia ullum e primis curialibus beneficiis. Ille nobis viginti quinque amplos caules capitatos in munus obtulit, 125 multo sane meliores quam qui in Gallia proveniunt; illius vicinus, tres naporum modios; atque ita de cæteris.

14. Je vous assure que Québec est bien situé et que la nature et la condition du pays environnant sont des plus heureuses. Trois-Rivières, à trente lieues de distance, est beaucoup plus riche et prospère. Et Montréal les dépasse en fertilité. Même le pays des Hurons les surpasserait encore, s'il n'était infesté par les Iroquois avec lesquels ils sont en guerre. Le ciel y est plus chaud et par conséquent plus productif. Nous avons l'espoir qu'avec la faveur et le secours de Dieu, et aussi grâce à la sagesse et à l'énergie de notre Gouverneur, qui a emmené grand nombre de soldats, on les forcera à faire la paix. Autrement, ils devront bien se préparer à la guerre.

14. Sitam felicem Quebecci et omnis circumiacentis regionis natura et conditionem prædico; Tres Fluvii triginta leucis dissiti longe uberiores sunt multoque beatiores; atque ipsis Mons Regius fertilior.

15 Il s'agit ici, sans doute, de Jean Le Sueur, abbé de Saint-Sauveur, ami de Jean Bourdon (DBC, I:482-483). Ce passage pourrait indiquer que Simon Denys et sa famille ont d'abord été logés à la côte Sainte-Geneviève, où l'abbé et Jean Bourdon demeuraient. 
130 Etiam Huronum regio melior, nisi eam Hyroquæi quibuscum ipsis bellum est eam infestarent. Cœli tractus calidior ac proinde feracior. In eam spem ingredimur fore ut Deo, et consiliis et conatibus Præfecti nostri, qui secum milites multos advexit, favente et aspirante, pacem inire cogantur; alioquin ad bellum se parent.

\section{4 alioquin corr. de alioqui}

15. Ma femme et moi, nous nous plaisons tellement dans ce pays que, si quelque force ne nous en chasse, nous avons bien l'intention d'y achever notre vie, sans renoncer toutefois à l'espérance de revoir la France, lorsque notre demeure sera établie et toutes nos affaires mises en ordre. Car si l'on traite les hommes avec autant d'égards, c'est mieux encore avec les filles. On les recherche en mariage avec tant d'empressement qu'on ne tient aucun compte de leur fortune, pourvu qu'elles soient sages. Si elles ne l'étaient pas, on les renverrait aussitôt. Ce châtiment est le seul dont on ait encore usé contre tous les crimes qui se sont commis ici ${ }^{16}$. Celui qui pourra employer six ouvriers pendant une année et demie vivra plus à l'aise que s'il avait en France un revenu de dix mille livres. Je ne voudrais pas vous laisser croire que $j$ 'exagère. Tous ceux qui débarquent ici trouveront que je dis la vérité. Même si je voulais mentir à quelqu'un, ce ne serait pas à vous. Vous me connaissez.

135 15. Coniugi meæ mihique adeo arridet hæcce regio ut, nisi quæ vis nos expellat, nobis statutum sit hic ætatem traducere, non tamen omni spe abiecta Galliæ revisendæ, ubi nostrum hic domicilium stabiliverimus resque omnes nostras composuerimus. Enimvero, si tam præclare cum viris, melius etiam cum puellis agitur. Ita ambiuntur

140 ad connubia, ut earum facultatum nulla ratio habeatur, modo sapientes sint; sin minus, subito remitterentur: quæ una animadversio adhuc adhibita est adversus omnia crimina quæ hic patrata sunt. Qui sex operarios hic poterit occupare, post sesqui-annum beatius vivet quam si ipsi in Gallia decem millia nummum redirent. Ne me quid145 quam augendæ rei causa dicere existimes velim. Quotquot huc appellent me veracem comperient. Si cuiquam mortalium mendax essem, at tibi mentiri nollem. Me nosti.

\section{6 hic aj. sur ligne}

16. En plus de tous ces avantages dont j'ai parlé, il y en a trois ${ }^{17}$ que des hommes industrieux ne mépriseront pas. Les

${ }^{16}$ Animadversio signifie une exécution capitale. On peut donc négliger les quelques condamnations au chevalet qui avaient eu lieu (Journal des Jésuites, 23, 35, 106). Cela admis, l'affirmation n'est pas tout à fait exacte. Il y avait eu deux exécutions capitales en janvier et février 1649 (ibid., 120). Le P. Jérôme Lalemant signalait qu'elles étaient les deux premières dans la colonie, fixant sans doute le début de la colonie en 1632 .

${ }^{17}$ Le texte de Denys n'énumère que deux de ces avantages: les mines et la pêche aux marsouins. A moins que l'espérance d'une pacification de l'Acadie puisse être comptée comme un troisième. 
mines de fer, de plomb et d'étain laissent espérer beaucoup de fruit et de profit. La pêche des marsouins blancs également, qui sont en grande abondance, car de certains d'entre eux on peut tirer trois barils d'huile. Il y a peu de temps, un de mes amis, qui habite le pays, en a pêché sept ou huit en dix ou douze jours et il m'a demandé de former une compagnie avec lui, à qui j'avais montré le moyen de les prendre en plus grand nombre. Plusieurs font pression sur nous pour être admis dans cette société et avoir part à cette capture. Notre Gouverneur espère que les affaires d'Acadie vont être arrangées et qu'on y donnera enfin une solution heureuse. De tous ces projets, il y en aura sûrement l'un ou l'autre qui arrivera à bonne fin. Le secours que j'attends de France l'an prochain décidera de toute l'affaire.

16. Præter omnia commoda quæ percensui, sunt et alia tria, viris industriis non contemnenda. Ferri, plumbi et stanni fodinæ multum

150 utilitatis et quæstus parere possunt. Tursionum alborum, quorum est magna copia, piscatio, cum ex quibusdam tursionibus tres olei cadi possit confici. Non ita pridem, familiaris quidam meus, unus ex incolis, intra decem aut duodecim dies, septem aut octo piscatus est; ut cum ipso societatem ineam rogavit me, cui multorum capiendorum

155 industriam aperui. Multi, ut in prædæ partem eamdemque societatem admittantur, contendunt a nobis. Præfectus noster sperat Acadiæ res compositum iri remque felicem exitum sortituram aliquando. [312] Ex iis omnibus consiliis aliquod ad exitum dies auspicato perducet. Galliæ auxilium quod in annum proxime sequentem expecto rem 160 totam concludet.

17. Je mets une fin à ma lettre; malgré moi, certes, car je n'ai pas encore fini de vous raconter le tout.

Je suis votre très humble et très dévoué serviteur et frère, Denys.

A Monsieur, monsieur du Brueil, sieur de Lagagnerie, dans sa maison, au quartier des Franciscains, à Tours.

17. Finem scribendi facio, invitus quidem, cum etiam non sit mihi omnia ad te perscribendi. Sum tuus humillimus et obsequentissimus servus et frater,

Dyonisius.

165 Domino, domino du Brueil, domino de Lagagnerie, suis in ædibus e regione Franciscanorum. Turonibus. 